Article

\title{
A Hydrodynamical Model for Carriers and Phonons With Generation-Recombination, Including Auger Effect
}

\author{
Alberto Rossani \\ Dipartimento di Scienza applicata e Tecnologia, Politecnico di Torino, Corso Duca degli Abruzzi 24, \\ Torino, 10129, Italy; E-Mail: alberto.rossani@ polito.it
}

Academic Editor: Kevin H. Knuth

Received: 6 June 2015 / Accepted: 28 August 2015 / Published: 9 September 2015

\begin{abstract}
The asymptotic procedure proposed allows to derive closed hydrodynamical equations from the kinetic equations of carriers and phonons (treated as a partecipating species) in a photon background. The direct generation-recombination processes are accounted for. The fluid-dynamical equations constructed for the chemical potentials of carriers, temperature, and drift velocity, are related to the extended thermodynamical (ET) ones for the chemical potentials of carriers, temperature, and drift velocity. In the drift-diffusion approximation the constitutive laws are derived and the Onsager relation recovered.
\end{abstract}

Keywords: carriers and phonons; macroscopic equations; generation-recombination

\section{Introduction}

In bipolar devices an interacting population of positively charged carriers (holes) must be taken into account, besides electrons and phonons. The hole-phonon interactions, similarly to electron-phonon, are emission/absobption phenomena.

Several generation-recombination (GR) events occur in semiconductors. In the presence of a photon background the most important ones are the radiative GR events. In the direct GR events a photon (pt) interact with a valence band electron and a couple electron-hole (e-h)is created:

$$
e+h \rightleftharpoons p t
$$

If the intensity of the photon field is low we must consider the Auger effect [1], which consists of two different processes and their inverse 
(a) electron capture: an electron fills an hole. The resulting energy is absorbed by another electron , and vice versa

$$
e+e+h \rightleftharpoons e^{*}
$$

where $*$ means "more energetic".

(b) hole capture: an hole combine with an electron. The resulting energy is absorbed by another hole, and vice versa

$$
h+h+e \rightleftharpoons h^{*}
$$

We start with the Bloch-Boltzmann-Peierls coupled kinetic equations for the distribution functions of carriers and phonons. A small parameter $\epsilon$ is introduced to account for the umklapp processes and both the interaction kernels and the distribution functions are expanded with respect to $\epsilon$. The lowest order equations show that the drifted maxwellian approximation is justified.

A hydrodynamical model, whose equations are similar to the ET ones [2], is then constructed for the temperature $T$ and the drift velocity $\mathbf{V}$ of the system, in addition to the chemical potentials $\mu_{e}, \mu_{h}$ of electrons and holes. Here such a model is not based on the Maximum Entropy Principle, like in ET, but strictly on kinetic theory.

The calculation of the source terms due to GR events takes advantage from the smallness of the GR collision frequencies

In the drift-diffusion approximation the constitutive laws are derived and the Onsager symmetry relationships verified.

We stress that in the present model

(1) The displaced Maxwellians approximation is not an ad hoc assumption but is justified by the expansion we apply

(2) Phonons are treated as a partecipating species, which brings energy and momentum

(3) The correct Phonon-phonon, carrier-phonon, and GR interaction kernels are utilized: we avoid the use of the relaxation time approximation.

The most qualifying point is (2). In fact, the usual assumption that the phonon field can be treated as a fixed background is dropped here, since "any thermal gradient give rise to transport of heat by the phonons, whilst an electric current, thought carried by electrons, cannot fail to transfer some of its momentum to the lattice vibrations, and drag them along with it" [3]. The present model can be seen as a generalization of a previous one [4] (with generation-recombination, more mathematical oriented), by means of the explicit treatment of phonons.

\section{Kinetic Equations}

Consider three interacting populations: electrons (e), holes (h), and phonons (pn). Moreover a background of photons ( $\mathrm{pt})$ is present. Let $N_{p n}^{g}(\mathbf{k}, \mathbf{x}, t)$ be the distribution function of phonons [quasi-momentum $\mathbf{k}$, energy $\omega_{p n}^{g}(\mathbf{k})$ ] of type $g$ (i.e., branch $g$ of the phonon spectrum) and $n_{\alpha}=$ 
$n_{\alpha}(\mathbf{p}, \mathbf{x}, t)$ the distribution function of carriers $\alpha=e, h$ (quasi-momentum $\mathbf{p}$, energy $\mathcal{E}_{\alpha}$ ). The kinetic equations for carriers and phonons read

$$
\begin{aligned}
\mathcal{D}_{e} n_{e} & =\left(\frac{\partial n_{e}}{\partial t}\right)_{e p n}+\left(\frac{\partial n_{e}}{\partial t}\right)_{e h} \\
\mathcal{D}_{h} n_{h} & =\left(\frac{\partial n_{h}}{\partial t}\right)_{h p n}+\left(\frac{\partial n_{h}}{\partial t}\right)_{h e} \\
\mathcal{D}_{p n}^{g} N_{p n}^{g} & =\left(\frac{\partial N_{p n}^{g}}{\partial t}\right)_{p n p n}+\left(\frac{\partial N_{p n}^{g}}{\partial t}\right)_{p n e}+\left(\frac{\partial N_{p n}^{g}}{\partial t}\right)_{p n h},
\end{aligned}
$$

where

$$
\begin{aligned}
\mathcal{D}_{\alpha} & =\frac{\partial}{\partial t}+\mathbf{v}_{\alpha} \cdot \frac{\partial}{\partial \mathbf{x}}+\lambda_{\alpha} e \mathbf{E} \cdot \frac{\partial}{\partial \mathbf{p}}, \quad \alpha=e, h, \quad \lambda_{\alpha}=-1,1 \\
\mathcal{D}_{p n}^{g} & =\frac{\partial}{\partial t}+\mathbf{u}_{p n}^{g} \cdot \frac{\partial}{\partial \mathbf{x}}
\end{aligned}
$$

with

$$
\mathbf{v}_{\alpha}=\frac{\partial \mathcal{E}_{\alpha}}{\partial \mathbf{p}}, \quad \mathbf{u}_{p n}^{g}=\frac{\partial \omega_{p n}^{g}}{\partial \mathbf{k}} .
$$

Observe that, being $\mathcal{E}_{\alpha}(-\mathbf{p})=\mathcal{E}_{\alpha}(\mathbf{p})$ and $\omega_{p n}^{g}(-\mathbf{k})=\omega_{p n}^{g}(\mathbf{k})$, we have $\mathbf{v}_{\alpha}(-\mathbf{p})=-\mathbf{v}_{\alpha}(\mathbf{p})$ and $\mathbf{u}_{p n}^{g}(-\mathbf{k})=-\mathbf{u}_{p n}^{g}(\mathbf{k})$.

At the right hand sides of the equations for phonons we have

$$
\begin{aligned}
\left(\partial N_{p n} / \partial t\right)_{p n p n}= & \int\left[( 1 / 2 ) \sum _ { g _ { 1 } g _ { 2 } } w _ { p n p n } ( \mathbf { k } _ { 1 } , \mathbf { k } _ { 2 } \rightarrow \mathbf { k } ) \left(-N_{p n}^{g}\left(1+N_{p n}^{g_{1}}\right)\left(1+N_{p n}^{g_{2}}\right)\right.\right. \\
& \left.+\left(1+N_{p n}^{g}\right) N_{p n}^{g_{1}} N_{p n}^{g_{2}}\right)+\sum_{g_{1} g_{3}} w_{p n p n}\left(\mathbf{k}, \mathbf{k}_{1} \rightarrow \mathbf{k}_{3}\right)\left(-\left(1+N_{p n}^{g}\right)\left(1+N_{p n}^{g_{1}}\right) N_{p n}^{g_{3}}\right. \\
& \left.+N_{p n}^{g} N_{p n}^{g_{1}}\left(1+N_{p n}^{g_{3}}\right)\right] \frac{d \mathbf{k}_{1}}{8 \pi^{3}},
\end{aligned}
$$

where

$$
\mathbf{k}_{2}=\mathbf{k}-\mathbf{k}_{1}+\mathbf{b}\left(\mathbf{k}_{1}, \mathbf{k}_{2} \rightarrow \mathbf{k}\right), \quad \mathbf{k}_{3}=\mathbf{k}+\mathbf{k}_{1}+\mathbf{b}\left(\mathbf{k}, \mathbf{k}_{1} \rightarrow \mathbf{k}_{3}\right)
$$

(b is an appropriate vector belonging to the reciprocal lattice), which account for three-phonon processes:

$$
(g, \mathbf{k}) \rightleftharpoons\left(g_{1}, \mathbf{k}_{1}\right)+\left(g_{2}, \mathbf{k}_{2}\right),\left(g_{3}, \mathbf{k}_{3}\right) \rightleftharpoons(g, \mathbf{k})+\left(g_{1}, \mathbf{k}_{1}\right)
$$

Moreover

$$
\left(\partial N_{p n}^{g} / \partial t\right)_{p n \alpha}=2 \sum_{\alpha} \int w_{p n \alpha}\left(\mathbf{p} \rightarrow \mathbf{p}^{\prime}, \mathbf{k}\right)\left(n_{\alpha}\left(1-n_{\alpha}^{\prime}\right)\left(1+N_{p n}^{g}\right)-\left(1-n_{\alpha}\right) n_{\alpha}^{\prime} N_{p n}^{g}\right) \frac{d \mathbf{p}_{\gamma}}{8 \pi^{3}},
$$

where $\mathbf{p}^{\prime}=\mathbf{p}-\mathbf{k}+\mathbf{b}\left(\mathbf{p} \rightarrow \mathbf{p}^{\prime}, \mathbf{k}\right)$, is the difference between the number of phonons $\mathbf{k}$ emitted by electrons with any quasimomenta $\mathbf{p}$ and the number of phonons absorbed by electrons with any $\mathbf{p}^{\prime}$, where $\mathbf{b}$ is a vector of the reciprocal lattice appropriate to the present interaction.

For carriers we have

$$
\begin{aligned}
\left(\partial n_{\alpha} / \partial t\right)_{\alpha p n}= & \sum_{g} \int w_{\alpha p n}\left(\mathbf{p}^{\prime}, \mathbf{k} \rightarrow \mathbf{p}\right)\left(n_{\alpha}^{\prime}\left(1-n_{\alpha}\right) N_{p n}^{g}-\left(1-n_{\alpha}^{\prime}\right) n_{\alpha}\left(1+N_{p n}^{g}\right)\right) \\
& \left.+w_{\alpha p n}\left(\mathbf{p}^{\prime \prime} \rightarrow \mathbf{p}, \mathbf{k}\right)\left(n_{\alpha}^{\prime \prime}\left(1-n_{\alpha}\right)\left(1+N_{p n}^{g}\right)-n_{\alpha}\left(1-n_{\alpha}^{\prime \prime}\right) N_{p n}^{g}\right)\right] \frac{d \mathbf{k}}{8 \pi^{3}}
\end{aligned}
$$


where

$$
\mathbf{p}^{\prime}=\mathbf{p}-\mathbf{k}+\mathbf{b}\left(\mathbf{p}^{\prime}, \mathbf{k} \rightarrow \mathbf{p}\right), \quad \mathbf{p}^{\prime \prime}=\mathbf{p}+\mathbf{k}+\mathbf{b}\left(\mathbf{p}^{\prime \prime} \rightarrow \mathbf{p}, \mathbf{k}\right) .
$$

The first term corresponds to to processes with emission of a phonon having quasimomentum $\mathbf{k}$ by an electron having a given quasimomentum $\mathbf{p}$ and reverse processes. The second term corresponds to processes with absorption of a phonon by an electron with quasimomentum $\mathbf{p}$ and reverse processes.

The $w$ 's are transition probabilities which account for energy conservation and satisfy the following symmetry relations:

$$
w_{\text {pne }}\left(\mathbf{p} \rightarrow \mathbf{p}^{\prime}, \mathbf{k}\right)=w_{\text {epn }}\left(\mathbf{p} \rightarrow \mathbf{p}^{\prime}, \mathbf{k}\right)=w_{\text {epn }}\left(\mathbf{p}^{\prime}, \mathbf{k} \rightarrow \mathbf{p}\right)
$$

Consider now the carrier-phonon system in contact with a photon medium. Let $N_{p t}$ be a Planck's distribution function at the temperature $T_{p t}$ :

$$
N_{p t}=\frac{1}{\exp \left(\omega_{p t} / T_{p t}\right)-1}
$$

where $\omega_{p t}=c k_{p t}$ (c is the speed of light). The collision integrals for the GR interactions are given by

$$
\left(\frac{\partial n_{\alpha}}{\partial t}\right)_{\alpha \gamma}=2 \int w_{\alpha \gamma}\left(\mathbf{p}_{\alpha}, \mathbf{p}_{\gamma} \rightarrow \mathbf{k}_{p t}\right)\left[-n_{\gamma} n_{\alpha}\left(1+N_{p t}\right)+\left(1-n_{\gamma}\right)\left(1-n_{\alpha}\right) N_{p t}\right] \frac{d \mathbf{p}_{\gamma}}{8 \pi^{3}}
$$

where $\gamma=h, e$.

The transition probabilities account for energy conservation and satisfy the following symmetry relations:

$$
w_{e h}\left(\mathbf{p}_{e}, \mathbf{p}_{h} \rightarrow \mathbf{k}_{p t}\right)=w_{h e}\left(\mathbf{p}_{e}, \mathbf{p}_{h} \rightarrow \mathbf{k}_{p t}\right)
$$

The Auger GR contributions can be written [1] as

$$
\begin{aligned}
\left(\partial f_{e} / \partial t\right)_{A}= & \iiint G_{e}^{A}\left(\mathbf{k}, \mathbf{p}_{1}, \mathbf{p}_{2} \rightarrow \mathbf{k}\right) d \mathbf{k} d \mathbf{p}_{1} d \mathbf{p}_{2} \\
& -2 \iiint G_{e}^{A}\left(\mathbf{k}, \mathbf{p}, \mathbf{p}_{2} \rightarrow \mathbf{p}_{1}\right) d \mathbf{k} d \mathbf{p}_{1} d \mathbf{p}_{2}-\iiint G_{h}^{A}\left(\mathbf{p}, \mathbf{k}_{1}, \mathbf{k}_{2} \rightarrow \mathbf{k}\right) d \mathbf{k} d \mathbf{k}_{1} d \mathbf{k}_{2}, \\
\left(\partial f_{h} / \partial t\right)_{A}= & \iiint G_{h}^{A}\left(\mathbf{p}, \mathbf{k}_{1}, \mathbf{k}_{2} \rightarrow \mathbf{k}\right) d \mathbf{p} d \mathbf{k}_{1} d \mathbf{k}_{2} \\
& -2 \iiint G_{h}^{A}\left(\mathbf{p}, \mathbf{k}, \mathbf{k}_{2} \rightarrow \mathbf{k}_{1}\right) d \mathbf{p} d \mathbf{k}_{1} d \mathbf{k}_{2}-\iiint G_{e}^{A}\left(\mathbf{k}, \mathbf{p}_{1}, \mathbf{p}_{2} \rightarrow \mathbf{p}\right) d \mathbf{p} d \mathbf{p}_{1} d \mathbf{p}_{2},
\end{aligned}
$$

where

$$
\begin{aligned}
G_{e}^{A}\left(\mathbf{k}, \mathbf{p}_{1}, \mathbf{p}_{2} \rightarrow \mathbf{k}\right)= & W_{e}\left(\mathbf{k}, \mathbf{p}_{1}, \mathbf{p}_{2} \rightarrow \mathbf{k}\right)\left(f_{h}(\mathbf{k}) f_{e}\left(\mathbf{p}_{1}\right) f_{e}\left(\mathbf{p}_{2}\right)\left(1-f_{e}(\mathbf{p})\right)\right. \\
& -\left(1-f_{h}(\mathbf{k})\right)\left(1-f_{e}\left(\mathbf{p}_{2}\right)\left(1-f_{e}\left(\mathbf{p}_{1}\right) f_{e}(\mathbf{p})\right)\right. \\
G_{h}^{A}\left(\mathbf{p}, \mathbf{k}_{1}, \mathbf{k}_{2} \rightarrow \mathbf{k}\right)= & U_{h}\left(\mathbf{p}, \mathbf{k}_{1}, \mathbf{k}_{2} \rightarrow \mathbf{k}\right)\left(f_{e}(\mathbf{p}) f_{h}\left(\mathbf{k}_{1}\right) f_{h}\left(\mathbf{k}_{2}\right)\left(1-f_{h}(\mathbf{k})\right)\right. \\
& -\left(1-f_{e}(\mathbf{p})\right)\left(1-f_{h}\left(\mathbf{k}_{1}\right)\right)\left(1-f_{h}\left(\mathbf{k}_{2}\right) f_{h}(\mathbf{k})\right),
\end{aligned}
$$

where $W_{e}$ and $U_{h}$ are the Waldmann kernels of Auger processes, for electrons and holes, respectively. We consider now a system, exact but not closed, of four balance equations, to be utilized later. By projecting the equation for particles $\alpha$ over 1 we have :

$$
\frac{\partial \mathcal{N}_{\alpha}}{\partial t}+\lambda_{\alpha}(1 / e) \nabla \cdot \mathbf{J}_{\alpha}=Q_{0}^{G R}
$$


where the carrier number density $\left(\mathcal{N}_{\alpha}\right)$ and the electric currents $\mathbf{J}_{\alpha}$ are given by

$$
\mathcal{N}_{\alpha}=2 \int n_{\alpha} \frac{d \mathbf{p}}{8 \pi^{3}}, \quad \mathbf{J}_{\alpha}=2 \lambda_{\alpha} e \int \mathbf{v}_{\alpha} n_{\alpha} \frac{d \mathbf{p}}{8 \pi^{3}} .
$$

Moreover $Q_{0}^{G R}=Q_{R}^{0}+Q_{A}^{0}$, where we separated the radiative (R) and Auger (A) effects. By projecting the equations for particles $\alpha$ over $2 \mathbf{p}$ and the phonon ones on $\mathbf{k}$, summation gives the following balance equation for momentum:

$$
\begin{aligned}
\frac{\partial \mathbf{P}}{\partial t}+\nabla \cdot \mathbb{F}_{\mathbf{P}}= & -e \mathbf{E}\left(\mathcal{N}_{e}-\mathcal{N}_{h}\right)+2 \sum_{\alpha} \int\left(\frac{\partial n_{\alpha}}{\partial t}\right)_{\alpha p n} \frac{\mathbf{p} d \mathbf{p}}{8 \pi^{3}} \\
& +\sum_{g} \int\left[\left(\frac{\partial N_{p n}^{g}}{\partial t}\right)_{p n p n}+\sum_{\alpha} \int\left(\frac{\partial N_{p n}^{g}}{\partial t}\right)_{p n \alpha}\right] \frac{\mathbf{k} d \mathbf{k}}{8 \pi^{3}}+\mathbf{S}^{G R}
\end{aligned}
$$

where

$$
\begin{aligned}
\mathbf{P} & =2 \sum_{\alpha} \int n_{\alpha} \frac{\mathbf{p} d \mathbf{p}}{8 \pi^{3}}+\sum_{g} \int N_{p n}^{g} \frac{\mathbf{k} d \mathbf{k}}{8 \pi^{3}} \\
\mathbb{F}_{\mathbf{P}} & =2 \sum_{\alpha} \int n_{\alpha} \mathbf{v}_{\alpha} \otimes \mathbf{p} \frac{d \mathbf{p}}{8 \pi^{3}}+\sum_{g} \int N_{p n}^{g} \mathbf{u}_{p n}^{g} \otimes \mathbf{k} \frac{d \mathbf{k}}{8 \pi^{3}}
\end{aligned}
$$

Moreover $\mathbf{S}^{G R}=\mathbf{S}_{R}+\mathbf{S}_{A}$.

Finally, by projecting the equations for particles $\alpha$ over $2 \mathcal{E}_{\alpha}$ and the phonon ones over $\omega_{p n}^{g}$, summation gives the following balance equation for energy :

$$
\frac{\partial W}{\partial t}+\nabla \cdot \mathbf{F}_{W}=\left(\mathbf{J}_{e}+\mathbf{J}_{h}\right) \cdot \mathbf{E}+Q_{1}^{G R},
$$

where the energy density $W$ and the energy flux $\mathbf{F}_{W}$ are given by

$$
W=2 \sum_{\alpha} \int \mathcal{E}_{\alpha} n_{\alpha} \frac{d \mathbf{p}}{8 \pi^{3}}+\sum_{g} \int \omega_{p n}^{g} N_{p n}^{g} \frac{d \mathbf{k}}{8 \pi^{3}}
$$

and

$$
\mathbf{F}_{W}=2 \sum_{\alpha} \int \mathbf{v}_{\alpha} \mathcal{E}_{\alpha} n_{\alpha} \frac{d \mathbf{p}}{8 \pi^{3}}+\sum_{g} \int \mathbf{u}_{p n}^{g} \omega_{p n}^{g} N_{p n}^{g} \frac{d \mathbf{k}}{8 \pi^{3}},
$$

Moreover $Q_{1}^{G R}=Q_{R}^{1}+Q_{A}^{1}$

The source terms $Q_{R}^{0}, Q_{R}^{1}, \mathbf{S}_{R}$ due to radiative GR events are given by

$$
\begin{aligned}
Q_{R}^{\ell} & \left.=4 \iint w_{e h}\left(\mathbf{p}_{e}, \mathbf{p}_{h} \rightarrow \mathbf{k}_{p t}\right)\right]\left[-n_{h} n_{e}\left(1+N_{p t}\right)+\left(1-n_{h}\right)\left(1-n_{e}\right) N_{p t}\right]\left[\mathcal{E}_{e}\left(\mathbf{p}_{e}\right)+\mathcal{E}_{h}\left(\mathbf{p}_{h}\right)\right]^{\ell} \frac{d \mathbf{p}_{e} d \mathbf{p}_{h}}{64 \pi^{6}} \\
\mathbf{S}_{R} & \left.=4 \iint w_{e h}\left(\mathbf{p}_{e}, \mathbf{p}_{h} \rightarrow \mathbf{k}_{p t}\right)\right]\left[-n_{h} n_{e}\left(1+N_{p t}\right)+\left(1-n_{h}\right)\left(1-n_{e}\right) N_{p t}\right]\left(\mathbf{p}_{e}+\mathbf{p}_{h}\right) \frac{d \mathbf{p}_{e} d \mathbf{p}_{h}}{64 \pi^{6}}
\end{aligned}
$$

Moreover, the contribution of Auger effect reads

$$
Q_{A}^{\ell}=Q_{\ell}^{A e}+Q_{\ell}^{A h}
$$

where

$$
\begin{aligned}
Q_{\ell}^{A} & =\int\left(\partial f_{e} / \partial t\right)_{A}\left(\mathcal{E}_{e}+\mathcal{E}_{h}\right)^{\ell} d \mathbf{p}_{e} \\
\mathbf{S}^{A \ell} & =\int\left(\partial f_{e} / \partial t\right)_{A}\left(\mathbf{p}_{e}+\mathbf{p}_{h}\right) d \mathbf{p}_{e}
\end{aligned}
$$




\section{Asymptotic Expansion and Hydrodynamical Equations}

By following ref. [5], we expand both the interaction kernels and the distribution function with respect to a small parameter $\epsilon$ which accounts for umklapp (U) processes (which do not conserve momentum) in addition to normal $(\mathrm{N})$ processes (which conserve momentum).

We start with carriers (the extension to phonons is trivial). The singular expansion for $w_{\alpha p n}$ reads

$$
w_{\alpha p n}=(1 / \epsilon) w_{\alpha p n}^{N}+w_{\alpha p n}^{U} .
$$

The sought expansions for $n_{\alpha}$ and $N_{p n}^{g}$ read

$$
n_{\alpha}=n_{\alpha}^{N}+\epsilon n_{\alpha}^{U}, \quad N_{p n}^{g}=N_{p t}^{g N}+\epsilon N_{p n}^{g U} .
$$

According to [3], at low temperatures $\epsilon$ decreases exponentially as $\exp \left(-\mathcal{C} T_{D} / T\right)$, where $T$ is a characteristic temperature of the system, $T_{D}$ is the Debye temperature, and $\mathcal{C}$ is of the order of unity. Therefore we can say that the present expansion is valid for $T<<T_{D}$.

We can write now

$$
\left(\frac{\partial n_{\alpha}}{\partial t}\right)_{\alpha p n}=\left(\frac{\partial n_{\alpha}}{\partial t}\right)_{\alpha p n}^{N}+\epsilon\left(\frac{\partial n_{\alpha}}{\partial t}\right)_{\alpha p n}^{U}
$$

where

$$
\begin{aligned}
& \left(\frac{\partial n_{\alpha}}{\partial t}\right)_{\alpha p n}^{N}=(1 / \epsilon)\left(\frac{\partial n_{\alpha}}{\partial t}\right)_{\alpha p n}^{N N}+\left(\frac{\partial n_{e}}{\partial t}\right)_{\alpha p n}^{N U} \\
& \left(\frac{\partial n_{\alpha}}{\partial t}\right)_{\alpha p n}^{U}=(1 / \epsilon)\left(\frac{\partial n_{\alpha}}{\partial t}\right)_{\alpha p n}^{U N}+\left(\frac{\partial n_{e}}{\partial t}\right)_{\alpha p n}^{U U}
\end{aligned}
$$

By collecting all these terms we have

$$
\mathcal{D}_{\alpha} n_{\alpha}^{N}-\left(\frac{\partial n_{\alpha}}{\partial t}\right)_{G R}^{N}=(1 / \epsilon)\left(\frac{\partial n_{\alpha}}{\partial t}\right)_{\alpha p n}^{N N}+\left(\frac{\partial n_{\alpha}}{\partial t}\right)_{\alpha p n}^{N U}+\left(\frac{\partial n_{\alpha}}{\partial t}\right)_{\alpha p n}^{U N},
$$

where we neglected the terms of order $\epsilon^{1}$.

At the orders $\epsilon^{-1}$ and $\epsilon^{0}$ we get

$$
\begin{aligned}
\left(\frac{\partial n_{\alpha}}{\partial t}\right)_{\alpha p n}^{N N} & =0 \\
\mathcal{D}_{\alpha} n_{\alpha}-\left(\frac{\partial n_{\alpha}}{\partial t}\right)_{G R}^{N} & =\left(\frac{\partial n_{\alpha}}{\partial t}\right)_{\alpha p n}^{N U}+\left(\frac{\partial n_{\alpha}}{\partial t}\right)_{\alpha p n}^{U N}
\end{aligned}
$$

Analogously, for phonons

$$
\begin{aligned}
& \left(\frac{\partial N_{p n}^{g}}{\partial t}\right)_{p n p n}^{N N}+\sum_{\alpha}\left(\frac{\partial N_{p n}^{g}}{\partial t}\right)_{p n \alpha}^{N N}=0 \\
& \mathcal{D}_{p n}^{g} N_{p n}^{g N}=\sum_{\alpha}\left[\left(\frac{\partial N_{p n}^{g}}{\partial t}\right)_{\alpha p n}^{N U}+\left(\frac{\partial N_{p n}^{g}}{\partial t}\right)_{\alpha p n}^{U N}\right]+\left(\frac{\partial N_{p n}^{g}}{\partial t}\right)_{p n p n}^{N U} .
\end{aligned}
$$

By taking into account momentum (it is a $N$ - process) and energy conservation, the equations of order $\epsilon^{-1}$ are solved (see Appendix) by

$$
n_{\alpha}^{N}=\mathcal{F}\left[\left(\mathcal{E}_{\alpha}-\mathbf{V} \cdot \mathbf{p}-\mu_{\alpha}\right) / T\right], \quad N_{p n}^{g N}=\mathcal{B}\left[\left(\omega_{p n}^{g}-\mathbf{V} \cdot \mathbf{k}\right) / T\right],
$$


where

$$
\mathcal{F}(\zeta)=\frac{1}{e^{\zeta}+1}, \quad \mathcal{B}(\zeta)=\frac{1}{e^{\zeta}-1}
$$

that is the drifted Fermi-Dirac (FD) and Bose-Einstein (BE) distribution functions.

The meaning of $\mathbf{V}$ is simple. Let $\hat{\mathbf{v}}_{\alpha}$ the most probable velocity of carriers $\alpha$, given by

$$
\frac{\partial}{\partial \mathbf{p}}\left(\mathcal{E}_{\alpha}-\mathbf{V} \cdot \mathbf{p}\right)=\mathbf{0}
$$

It is easily seen that $\hat{\mathbf{v}}_{\alpha}=\mathbf{V}$. Analogously, for phonons, $\hat{\mathbf{u}}_{p n}^{g}=\mathbf{V}$.

The distribution functions are usually expanded as follows

$$
\begin{aligned}
n_{\alpha}^{N} & =\mathcal{F}\left[\left(\mathcal{E}_{\alpha}-\mu_{\alpha}\right) / T\right]-\beta \mathbf{V} \cdot \mathbf{p} \mathcal{F}^{\prime}\left[\left(\mathcal{E}_{\alpha}-\mu_{\alpha}\right) / T\right]=n_{\alpha}^{0}+n_{\alpha}^{1} \\
N_{p n}^{g N} & =\mathcal{B}\left(\omega_{p n}^{g} / T\right)-\beta \mathbf{V} \cdot \mathbf{k} \mathcal{B}^{\prime}\left(\omega_{p n}^{g} / T\right)=N_{g}^{0}+N_{g}^{1},
\end{aligned}
$$

where

$$
\mathcal{F}^{\prime}(\zeta)=-\mathcal{F}^{2}(\zeta) e^{\zeta}, \mathcal{B}^{\prime}(\zeta)=-\mathcal{B}^{2}(\zeta) e^{\zeta}
$$

This simplification is valid when the drift energy is small compared to thermal energy. Under this assumption, after some calculations we find

$$
\begin{aligned}
\left(\partial N_{p n}^{g} / \partial t\right)_{p p}^{N U}= & \beta \mathbf{V} \cdot\left\{\int \left[(1 / 2) \sum_{g_{1} g_{2}}\left(1+N_{p n}^{g 0}\right) N_{p n}^{g 0} N_{p n}^{g_{2} 0} w_{p n p n}^{U}\left(\mathbf{k}_{1}, \mathbf{k}_{2} \rightarrow \mathbf{k}\right)\left(\mathbf{k}_{2}+\mathbf{k}_{1}-\mathbf{k}\right)\right.\right. \\
& \left.\left.+\sum_{g_{1} g_{3}}\left(1+N_{p n}^{g_{3} 0}\right) N_{p n}^{g 0} N_{p n}^{g_{1} 0} w_{p n p n}^{U}\left(\mathbf{k}, \mathbf{k}_{1} \rightarrow \mathbf{k}_{3}\right)\left(\mathbf{k}_{3}-\mathbf{k}_{1}-\mathbf{k}\right)\right] \frac{d \mathbf{k}_{1}}{8 \pi^{3}}\right\} \\
\left(\partial N_{p n}^{g} / \partial t\right)_{p n \alpha}^{N U}= & \beta \mathbf{V} \cdot\left\{2 \int\left(1-n_{\alpha}^{\prime 0}\right) n_{\alpha}^{0}\left(1+N_{p n}^{g_{1} 0}\right) w_{p n \alpha}^{U}\left(\mathbf{p} \rightarrow \mathbf{p}^{\prime}, \mathbf{k}\right)\left(\mathbf{p}-\mathbf{k}-\mathbf{p}^{\prime}\right) \frac{d \mathbf{p}}{8 \pi^{3}}\right\}
\end{aligned}
$$

and

$$
\begin{aligned}
\left(\partial n_{\alpha} / \partial t\right)_{\alpha p n}^{N U}= & \beta \mathbf{V} \cdot \sum_{g}\left\{\int\left(1-n_{\mathbf{p}}^{0}\right) n_{\alpha}^{\prime 0} N_{p n}^{g 0} w_{\alpha p n}^{U}\left(\mathbf{p}^{\prime}, \mathbf{k} \rightarrow \mathbf{p}\right)\left(\mathbf{k}+\mathbf{p}^{\prime}-\mathbf{p}\right)\right. \\
& \left.+\left(1-n_{\alpha}^{0}\right) n_{\alpha}^{\prime 0}\left(1+N_{p n}^{g 0}\right) w_{\alpha p n}^{U}\left(\mathbf{p}^{\prime} \rightarrow \mathbf{p}, \mathbf{k}\right)\left(\mathbf{p}^{\prime}-\mathbf{k}-\mathbf{p}\right)\right\} \frac{d \mathbf{k}}{8 \pi^{3}}
\end{aligned}
$$

Starting from the equations of order $\epsilon^{0}$, a hydrodynamical model can be constructed now, related to ET one [2], for the temperature $T$ and the drift velocity $\mathbf{V}$ of the system, in addition to the chemical potentials $\mu_{e}, \mu_{h}$.

By projecting the equation for carrier $\alpha$ over 1, the balance equation for particles $\alpha$ reads

$$
\frac{\partial}{\partial t} \int n_{\alpha} d \mathbf{p}+\nabla \cdot \int \mathbf{v}_{\alpha} n_{\alpha} d \mathbf{p}=8 \pi^{3} Q_{0 N}^{G R}
$$

Hereinafter the subscript $N$ in the source terms means that in their definition we utilize $n_{\alpha}^{N}$ for the integration. 
By projecting the equations for carriers over $2 \mathbf{p}$ and the phonon ones on $\mathbf{k}$, summation gives the following balance equation for the total momentum:

$$
\begin{aligned}
& \frac{\partial}{\partial t}\left(2 \int \sum_{\alpha} n_{\alpha}^{1} \mathbf{p} d \mathbf{p}+\int \sum_{g} N_{g}^{1} \mathbf{k} d \mathbf{k}\right)+\nabla \cdot\left(2 \int \sum_{\alpha} n_{\alpha}^{0} \mathbf{v}_{\alpha} \otimes \mathbf{p} d \mathbf{p}+\int \sum_{g} N_{g}^{0} \mathbf{u}_{g} \otimes \mathbf{k} d \mathbf{k}\right) \\
= & 2 \mathbf{E} \sum_{\alpha} \lambda_{\alpha} \int n_{\alpha}^{0} d \mathbf{p}+2 \sum_{\alpha} \int\left(\frac{\partial n_{\alpha}}{\partial t}\right)_{\alpha p n}^{N U} \mathbf{p} d \mathbf{p} \\
& +\sum_{g} \int\left[\left(\frac{\partial N_{p n}^{g}}{\partial t}\right)_{p n p n}^{N U}+\sum_{\alpha}\left(\frac{\partial N_{p n}^{g}}{\partial t}\right)_{p n \alpha}^{N U}\right] \mathbf{k} d \mathbf{k}+\mathbf{S}_{N}^{G R} .
\end{aligned}
$$

where we took advantage of

$$
2 \int\left(\frac{\partial n_{\mathbf{p}}}{\partial t}\right)_{e p}^{U N} \mathbf{p} d \mathbf{p}+\sum_{g} \int\left[\left(\frac{\partial N_{p n}^{g}}{\partial t}\right)_{p n p n}^{U N}+\sum_{\alpha} \int\left(\frac{\partial N_{p n}^{g}}{\partial t}\right)_{p n \alpha}^{U N}\right] \mathbf{k} d \mathbf{k}=0
$$

due to momentum conservation for $\mathrm{N}$-processes.

Finally, by projecting the carrier equations over $2 \mathcal{E}_{\alpha}$ and the phonon ones over $\omega_{p n}^{g}$, summation gives the following energy balance equation:

$$
\begin{aligned}
& \frac{\partial}{\partial t}\left(2 \sum_{\alpha} \int \mathcal{E}_{\alpha} n_{\alpha}^{0} d \mathbf{p}+\sum_{g} \omega_{g} N_{g}^{0} d \mathbf{k}\right)+\nabla \cdot\left(2 \sum_{\alpha} \int \mathbf{v}_{\alpha} \mathcal{E}_{\alpha} n_{\alpha}^{1} d \mathbf{p}+\sum_{g} \int \mathbf{u}_{g} \omega_{g} N_{g}^{1} d \mathbf{k}\right) \\
= & \sum_{\alpha} \lambda_{\alpha} e \mathbf{E} \cdot \int \mathbf{v}_{\alpha} n_{\alpha}^{1} d \mathbf{p}+8 \pi^{3} Q_{1 N}^{G R} .
\end{aligned}
$$

\section{Source Terms}

The source terms are small quantities since the relaxation time $\tau_{R G}$ of the RG processes is much larger than the one $\left(\tau_{\alpha p n}\right)$ of the $\alpha-p n$ interactions [6]. Hence we shall utilize for their calculation an approximation which properly accounts for this smallness.

The equations of order $\epsilon^{0}$ can be written, after a suitable adimensionalization, as follows

$$
\begin{aligned}
& \mathcal{D}_{\alpha} n_{\alpha}^{N}-\left(\frac{\partial n_{\alpha}}{\partial t}\right)_{G R}^{N}=(1 / \eta)\left[\left(\frac{\partial n_{\alpha}}{\partial t}\right)_{\alpha p n}^{N U}+\left(\frac{\partial n_{\alpha}}{\partial t}\right)_{\alpha p n}^{U N}\right], \\
& \mathcal{D}_{p n}^{g} N_{p n}^{g N}=(1 / \eta)\left\{\sum_{\alpha}\left[\left(\frac{\partial N_{p n}^{g}}{\partial t}\right)_{\alpha p n}^{N U}+\left(\frac{\partial N_{p n}^{g}}{\partial t}\right)_{\alpha p n}^{U N}\right]+\left(\frac{\partial N_{p n}^{g}}{\partial t}\right)_{p n p n}^{N U}+\left(\frac{\partial N_{p n}^{g}}{\partial t}\right)_{p n p n}^{U N}\right\}
\end{aligned}
$$

where $\eta=\mathcal{O}\left(\tau_{\alpha p n} / \tau_{\alpha \gamma}\right)$ is a small parameter. At the orders $\eta^{-1}$ and $\eta^{0}$ we have, respectively

$$
\begin{gathered}
\left(\frac{\partial n_{\alpha}}{\partial t}\right)_{\alpha p n}^{N U}+\left(\frac{\partial n_{\alpha}}{\partial t}\right)_{\alpha p n}^{U N}=0 \\
\mathcal{D}_{\alpha} n_{\alpha}^{N}=\left(\frac{\partial n_{\alpha}}{\partial t}\right)_{G R}^{N}
\end{gathered}
$$

Analogously we have

$$
\begin{aligned}
& \left(\frac{\partial N_{p n}^{g}}{\partial t}\right)_{p n p n}^{N U}+\left(\frac{\partial N_{p n}^{g}}{\partial t}\right)_{p n p n}^{U N}+\sum_{\alpha}\left[\left(\frac{\partial N_{p n}^{g}}{\partial t}\right)_{p n \alpha}^{N U}+\left(\frac{\partial N_{p n}^{g}}{\partial t}\right)_{p n \alpha}^{U N}\right]=0 \\
& \mathcal{D}_{p n}^{g} N_{p n}^{g}=\left(\frac{\partial N_{p n}^{g}}{\partial t}\right)_{p n p h}^{N}
\end{aligned}
$$


By projecting the equations of order $\eta^{-1}$ for carriers over $2 \mathbf{p}$ and the phonon ones on $\mathbf{k}$, summation gives

$$
2 \sum_{\alpha} \int\left(\frac{\partial n_{\alpha}}{\partial t}\right)_{\alpha p}^{N U} \mathbf{p} d \mathbf{p}+\sum_{g} \int\left[\left(\frac{\partial N_{p n}^{g}}{\partial t}\right)_{p m p n}^{N U}+\sum_{\alpha}\left(\frac{\partial N_{\alpha}^{g}}{\partial t}\right)_{p n \alpha}^{N U}\right] \mathbf{k} d \mathbf{k}=0
$$

which, due to $(1,2,3)$, shows that in the present approximation $\mathbf{V}=\mathbf{0}$, so that $Q_{0}^{G R}, Q_{1}^{G R}$, are simply approximated by setting $n_{\alpha}=n_{\alpha}^{0}$ and $N_{p n}^{g}=N_{g}^{0}$. Moreover, symmetry arguments lead to $\mathbf{S}^{G R}=\mathbf{0}$.

In the low density approximation $[6]\left(1-n_{\alpha} \simeq 1\right)$ we can write

$$
n_{\alpha}^{0}=\mathcal{N}_{\alpha} C_{\alpha}(T) \exp \left(-\mathcal{E}_{\alpha} / T\right),
$$

where $\mathcal{N}_{\alpha}$ is the number density of particles $\alpha$ and

$$
C_{\alpha}(T)=\frac{4 \pi^{3}}{\int \exp \left(-\mathcal{E}_{\alpha} / T\right) d \mathbf{p}_{\alpha}},
$$

so that

$$
Q_{\ell}=4 \iint w_{e h}\left(\mathbf{p}_{e}, \mathbf{p}_{h} \rightarrow \mathbf{k}_{p t}\right)\left[\mathcal{E}_{e}\left(\mathbf{p}_{e}\right)+\mathcal{E}_{h}\left(\mathbf{p}_{h}\right)\right]^{\ell}\left\{\mathcal{N}_{e} \mathcal{N}_{h} C_{e} C_{h} \exp \left[\omega_{p t}\left(\beta_{p t}-\beta\right)\right]-1\right\} N_{p t} \frac{d \mathbf{p}_{e} d \mathbf{p}_{h}}{8 \pi^{6}}
$$

Observe that $Q_{0}$ and $Q_{1}$ depend linearly on $\mathcal{N}_{e} \mathcal{N}_{h}$, while

$$
Q_{A}^{\ell}=Q_{\ell}^{A e}+Q_{\ell}^{A h}
$$

where

$$
\begin{aligned}
G_{e}^{A}\left(\mathbf{k}, \mathbf{p}_{1}, \mathbf{p}_{2} \rightarrow \mathbf{k}\right)= & W_{e}\left(\mathbf{k}, \mathbf{p}_{1}, \mathbf{p}_{2} \rightarrow \mathbf{k}\right)\left(C_{h} C_{e}^{2} \mathcal{N}_{h} \mathcal{N}_{e}\right) \exp \left[-\mathcal{E}_{e}\left(\mathbf{p}_{1}\right)-\mathcal{E}_{e}\left(\mathbf{p}_{1}\right)\right] \\
& -C_{e} \mathcal{N}_{e} \exp \left(-\mathcal{E}_{e}(\mathbf{p})\right) \\
G_{h}^{A}\left(\mathbf{p}, \mathbf{k}_{1}, \mathbf{k}_{2} \rightarrow \mathbf{k}\right)= & U_{h}\left(\mathbf{p}, \mathbf{k}_{1}, \mathbf{k}_{2} \rightarrow \mathbf{k}\right)\left[C_{e} \mathcal{N}_{e} C_{h}^{2} \mathcal{N}_{h}^{2} \exp \left(-\mathcal{E}_{e}(\mathbf{p})-\mathcal{E}_{h}\left(\mathbf{k}_{1}\right)-\mathcal{E}_{h}\left(\mathbf{k}_{2}\right)\right]\right) \\
& -C_{h} \mathcal{N}_{h} \exp \left(-\mathcal{E}_{h}(\mathbf{k})\right),
\end{aligned}
$$

are cubic with respect the number densities.

\section{Revised Drift-Diffusion Approximation and Constitutive Laws}

In the drift-diffusion approximation [7,8] (and, in particular for bipolar devices, [9]), we assume that the total momentum of the mixture does not vary appreciably over the momentum relaxation time. From the momentum balance equation we get

$$
-2 \beta\left(\mathbb{R}_{2}+\mathbb{R}_{4}\right) \cdot \nabla T-2 e \mathbb{R}_{1} \cdot \mathbf{E}_{e}+2 e \mathbb{R}_{3} \cdot \mathbf{E}_{h}-\beta \mathbb{R}_{5} \cdot \nabla T=\mathbb{B} \cdot \mathbf{V}
$$

which gives $\mathbf{V}$, where $\mathbf{E}_{\alpha}=\mathbf{E}-\lambda_{\alpha}(1 / e) \nabla \mu_{\alpha}$ and

$$
\begin{aligned}
\mathbb{R}_{l} & =\int \mathcal{F}^{\prime}\left[\beta\left(\mathcal{E}_{\alpha}-\mu_{\alpha}\right)\right] \mathbf{p} \otimes \mathbf{v}_{\alpha} d \mathbf{p}, \quad \alpha=e, h, \quad l=1,3 \\
\mathbb{R}_{l} & =\int \mathcal{F}^{\prime}\left[\beta\left(\mathcal{E}_{\alpha}-\mu_{\alpha}\right)\right]\left(\mathcal{E}_{\alpha}-\mu_{\alpha}\right) \mathbf{p} \otimes \mathbf{v}_{\alpha} d \mathbf{p}=M_{l}-\mu_{\alpha} \mathbb{R}_{l-1}, \quad \alpha=e, h, \quad l=2,4 \\
\mathbb{R}_{5} & =\sum_{g} \int \mathcal{B}^{\prime}\left(\beta \omega_{p n}^{g}\right) \omega_{p n}^{g} \mathbf{k} \otimes \mathbf{u}_{p n}^{g} d \mathbf{k} .
\end{aligned}
$$


The tensor $\mathbb{B}$, can be written in the following symmetric form

$$
\begin{aligned}
\mathbb{B}= & -\sum_{g_{1} g_{2} g_{3}} \iint N_{p n}^{g_{2} 0} N_{p n}^{g_{3} 0}\left(1+N_{p n}^{g_{1} 0}\right) w_{p p}\left(\mathbf{k}_{2}, \mathbf{k}_{3} \rightarrow \mathbf{k}_{1}\right)\left(\mathbf{k}_{1}-\mathbf{k}_{2}-\mathbf{k}_{3}\right) \otimes\left(\mathbf{k}_{1}-\mathbf{k}_{2}-\mathbf{k}_{3}\right) \frac{d \mathbf{k}_{1} d \mathbf{k}_{2}}{16 \pi^{3}} \\
& -2 \sum_{\alpha} \sum_{g} \iint n_{\alpha}^{0}\left(1-n_{\alpha}^{\prime 0}\right)\left(1+N_{p n}^{g 0}\right) w_{\alpha p}\left(\mathbf{p} \rightarrow \mathbf{p}^{\prime}, \mathbf{k}\right)\left(\mathbf{p}-\mathbf{k}-\mathbf{p}^{\prime}\right) \otimes\left(\mathbf{p}-\mathbf{k}-\mathbf{p}^{\prime}\right) \frac{d \mathbf{p} d \mathbf{k}}{8 \pi^{3}} .
\end{aligned}
$$

By utilizing the drifted FD or BE distribution functions, the electrical $\left(\mathbf{J}_{\alpha}\right)$ and thermal $\left(\mathbf{U}_{\alpha}, \mathbf{U}_{p}\right)$ currents are given by

$$
\begin{aligned}
\mathbf{J}_{\alpha} & =\frac{\lambda_{\alpha} e}{4 \pi^{3}} \int \mathbf{v}_{\alpha} n_{\alpha} d \mathbf{p}=-\frac{\lambda_{\alpha} e \beta}{4 \pi^{3}} \int \mathbf{v}_{\alpha} \mathbf{V} \cdot \mathbf{p} \mathcal{F}^{\prime}\left[\beta\left(\mathcal{E}_{\alpha}-\mu_{\alpha}\right)\right] d \mathbf{p} \\
\mathbf{U}_{\alpha} & =\frac{1}{4 \pi^{3}} \int \mathbf{v}_{\alpha}\left(\mathcal{E}_{\alpha}-\mu_{\alpha}\right) n_{\alpha} d \mathbf{p}=-\frac{\beta}{4 \pi^{3}} \int \mathbf{v}_{\alpha} \mathbf{V} \cdot \mathbf{p}\left(\mathcal{E}_{\alpha}-\mu_{\alpha}\right) \mathcal{F}^{\prime}\left[\beta\left(\mathcal{E}_{\alpha}-\mu_{\alpha}\right)\right] d \mathbf{p} \\
\mathbf{U}_{p n} & =\frac{1}{8 \pi^{3}} \sum_{g} \int \omega_{p n}^{g} \mathbf{u}_{p n}^{g} N_{g} d \mathbf{k}=-\frac{\beta}{8 \pi^{3}} \sum_{g} \int \omega_{p n}^{g} \mathbf{u}_{p n}^{g} \mathbf{V} \cdot \mathbf{k} \mathcal{B}^{\prime}\left(\beta \omega_{g}\right) d \mathbf{k}
\end{aligned}
$$

Now, by introducing $\mathbf{V}$,

$$
\begin{aligned}
\mathbf{J}_{e} & =-\frac{e \beta}{4 \pi^{3}}\left[2 e\left(\mathbb{K}_{11} \cdot \mathbf{E}_{e}-\mathbb{K}_{13} \cdot \mathbf{E}_{h}\right)+\beta\left(2 \mathbb{K}_{12}+2 K_{14}+\mathbb{K}_{15}\right) \cdot \nabla T\right] \\
\mathbf{U}_{e} & =\frac{\beta}{4 \pi^{3}}\left[2 e\left(\mathbb{K}_{21} \cdot \mathbf{E}_{e}-\mathbb{K}_{23} \cdot \mathbf{E}_{h}\right)+\beta\left(2 \mathbb{K}_{22}+2 \mathbb{K}_{24}+\mathbb{K}_{25}\right) \cdot \nabla T\right] \\
\mathbf{J}_{h} & =\frac{e \beta}{4 \pi^{3}}\left[2 e\left(\mathbb{K}_{31} \cdot \mathbf{E}_{e}-\mathbb{K}_{33} \cdot \mathbf{E}_{h}\right)+\beta\left(2 \mathbb{K}_{32}+2 \mathbb{K}_{34}+\mathbb{K}_{35}\right) \cdot \nabla T\right] \\
\mathbf{U}_{h} & =\frac{\beta}{4 \pi^{3}}\left[2 e\left(\mathbb{K}_{41} \cdot \mathbf{E}_{e}-\mathbb{K}_{43} \cdot \mathbf{E}_{h}\right)+\beta\left(2 K_{42}+2 \mathbb{K}_{44}+\mathbb{K}_{45}\right) \cdot \nabla T\right] \\
\mathbf{U}_{p n} & =\frac{\beta}{4 \pi^{3}}\left[2 e\left(\mathbb{K}_{51} \cdot \mathbf{E}_{e}-\mathbb{K}_{53} \cdot \mathbf{E}_{h}\right)+\beta\left(2 K_{52}+2 \mathbb{K}_{54}+\mathbb{K}_{55}\right) \cdot \nabla T\right],
\end{aligned}
$$

where $\mathbb{K}_{l m}=\widetilde{\mathbb{R}}_{l} \cdot \mathbb{B}^{-1} \cdot \mathbb{R}_{m}(\sim$ means transpose $)$. Since $\mathbb{B}=\widetilde{\mathbb{B}}$, the following Onsager symmetry relation is in order:

$$
\mathbb{K}_{l m}=\widetilde{\mathbb{I}}_{m l} .
$$

The cross effects of $\mathbf{E}_{h}$ on $\mathbf{J}_{e}$ and of $\mathbf{E}_{e}$ on $\mathbf{J}_{h}$ in a drift-diffusion model are discussed in [10].

Moreover, we can calculate the energy flux by its very definition:

$$
\begin{aligned}
\mathbf{F}_{W} & =-\beta\left\{2 \sum_{\alpha} \int \mathbf{v}_{\alpha} \otimes \mathbf{p} \mathcal{E}_{\alpha} \mathcal{F}^{\prime}\left[\beta\left(\mathcal{E}_{\alpha}-\mu_{\alpha}\right)\right] \frac{d \mathbf{p}}{8 \pi^{3}}+\sum_{g} \int \mathbf{u}_{p n}^{g} \otimes \mathbf{k} \omega_{p n}^{g} \mathcal{B}^{\prime}\left(\beta \omega_{p n}^{g}\right) \frac{d \mathbf{k}}{8 \pi^{3}}\right\} \cdot \mathbf{V} \\
& =-\frac{\beta}{8 \pi^{3}}\left[2\left(\widetilde{M} M_{2}+\widetilde{M}_{4}\right)+\widetilde{\mathbb{R}}_{5}\right] \cdot \mathbf{V} .
\end{aligned}
$$

The system of the drift-diffusion equations is obtained by inserting $\mathbf{J}_{e}$ and $\mathbf{J}_{h}$ into the carrier balance equations and $\mathbf{F}_{W}$ into the energy balance one.

\section{Conclusions}

A new fluid-dynamical model for a carrier-phonon system in a photon background is proposed, whose equations are certainly related to ET ones, based on the maximum energy principle [11-14]. However 
here the treatment is based entirely on kinetic theory, with no need to adjust some free parameters, based on comparisons with Monte Carlo calculations. A revised drift-diffusion approximation has been derived, which include an energy balance equation. The fulfilment of the Onsager symmetry relations is not trivial, since it cannot taken for granted in many macroscopic models [15].

Finally we observe that the asymptotic expansion is valid $(\epsilon<<1)$ when the room temperature is much lower than the Debye one (in silicon, for example). Hence, proper candidates for applications are silicon devices.

\section{Appendix}

Consider the equations at order $\epsilon^{-1}$ :

$$
\begin{aligned}
\left(\frac{\partial n_{\alpha}}{\partial t}\right)_{e p}^{N N} & =0 \\
\left(\frac{\partial N_{p n}^{g}}{\partial t}\right)_{p p}^{N N}+\left(\frac{\partial N_{p n}^{g}}{\partial t}\right)_{p e}^{N N} & =0 .
\end{aligned}
$$

By following the same approach as in [5] it can be shown that these conditions are equivalent to

$$
\begin{aligned}
N_{p n}^{g N}\left(1+N_{p n}^{g_{2} N}\right)\left(1+N_{p n}^{g_{1} N}\right) & =\left(1+N_{p n}^{g N}\right)\left(1+N_{p n}^{g_{1} N}\right) N_{p n}^{g_{2} N} \forall \mathbf{k}, \mathbf{k}_{1} \\
n_{\alpha}^{N}(\mathbf{p})\left[1-n_{\alpha}^{N}\left(\mathbf{p}^{\prime}\right)\right]\left(1+N_{p n}^{g N}\right) & =n_{\alpha}^{N}\left(\mathbf{p}^{\prime}\right)\left[1-n_{\alpha}^{N}(\mathbf{p})\right] N_{p n}^{g N} \forall \mathbf{p}, \mathbf{k} .
\end{aligned}
$$

Condition (8) shows that $\ln \left[N_{p n}^{g N} /\left(1+N_{p n}^{g N}\right)\right]$ is a collisional invariant for phonons. In the case of $\mathrm{N}$-processes

$$
\ln \frac{N_{p n}^{g N}}{1+N_{p n}^{g N}}=\left(\mathbf{V} \cdot \mathbf{k}-\omega_{p n}^{g}\right) / T .
$$

By inserting (10) into (9), since

$$
\mathbf{p}=\mathbf{p}^{\prime}+\mathbf{k}, \quad \mathcal{E}_{\alpha}(\mathbf{p})=\mathcal{E}_{\alpha}\left(\mathbf{p}^{\prime}\right)+\omega_{p n}^{g}
$$

we find that

$$
\ln \frac{n_{\alpha}^{N}(\mathbf{p})}{1-n_{\alpha}^{N}(\mathbf{p})}+\left(\mathcal{E}_{\alpha}-\mathbf{V} \cdot \mathbf{p}\right) / T
$$

is a collisional invariant for $\alpha$-particles:

$$
\ln \frac{n_{\alpha}^{N}(\mathbf{p})}{1-n_{\alpha}^{N}(\mathbf{p})}=\left(-\mathcal{E}_{\alpha}+\mathbf{V} \cdot \mathbf{p}+\mu_{\alpha}\right) / T .
$$

\section{Conflicts of Interest}

The author declares no conflict of interest.

\section{References}

1. Rossani, A.; Spiga, G. Auger effect in the generalized kinetic theory of electrons and holes. $J$. Math. Phys. 2006, 47, 0133101. 
2. Anile, A.M.; Pennisi, S. Thermodynamic derivation of the hydrodynamical model for charge transport in semiconductors. Phys. Rev. B 1992, 46, 13186.

3. Ziman, J.M. Electrons and Phonons; Oxford: Claredon, UK, 1960.

4. Poupaud, F. On a system of nonlinear Boltzmann equations of semiconductor physics. SIAM J. Appl. Math. 1990, 50, 1593-1606.

5. Rossani, A. Generalized balance equations for an electron-phonon system. J. Phys. A Math. Theor. 2010, 43, 165002.

6. Wachutka, G.K. Rigorous Thermodynamic treatment of heat generation and conduction in semiconductor device modeling. IEEE Trans. Comput.-aided Des. Integr. Circuits Syst. 1990, 9, 1141-1149.

7. Ben Abdallah, N.; Degond, P.; Geyneis, S. An energy transport model for semiconductors derived from the Boltzmann equation. J. Stat. Phys. 1996, 84, 205-231.

8. Ben Abdallah, N.; Degond, P. On a hierarchy of macroscopic models for semiconductors. J. Math. Phys. 1996, 37, 3306-3333.

9. Markowich, P.A.; Ringhofer, C.A.; Schmeiser, C. Semiconductor equations; Springer: Wien, Austria, 1990.

10. Reznik, D.; Gerlach, W. Generalized drift-diffusion model of bipolar transport in semiconductors. Electr. Eng. 1996, 79, 219-225.

11. Romano, V.; Zwierz, M. Electron phonon hydrodynamical model for semiconductors. Z. Angew. Math Phys 2010, 61, 1111-1131.

12. Romano, V.; Rusakov, A. 2D numerical simulations of an electron-phonon hydrodynamical model based on the maximum energy principle. Comput. Methods Appl. Mech. Eng. 2010, 199, 2741-2751.

13. Ali', G.; Mascali, G.; Romano, V.; Torcasio, R.C. A hydrodynamical model for covalent semiconductors with a generalized dispersion relation. Eur. J. Appl. Math. 2014, 25, 255-276.

14. Mascali, G. A hydrodynamical model for silicon semiconductors including crystal heating. Eur. $J$. Appl. Math. 2015, 26, 477-449.

15. Muscato, $\mathrm{O}$. The Onsager reciprocity principle as a check of consistency for semiconductor carrier transport models. Physica A 2001, 289, 422-458.

(c) 2015 by the author; licensee MDPI, Basel, Switzerland. This article is an open access article distributed under the terms and conditions of the Creative Commons Attribution license (http://creativecommons.org/licenses/by/4.0/). 